\title{
Clock Gene Expression in the Murine Gastrointestinal Tract: Endogenous Rhythmicity and Effects of a Feeding Regimen
}

\author{
WILLEMIJNTJE A. HOOGERWERF, ${ }^{*}$ HELEN L. HELLMICH, ${ }^{\ddagger}$ GERMAINE CORNÉLISSEN, ${ }^{\S}$ FRANZ HALBERG,, \\ VAHAKN B. SHAHINIAN," JONATHON BOSTWICK, * TOR C. SAVIDGE," and VINCENT M. CASSONE\#

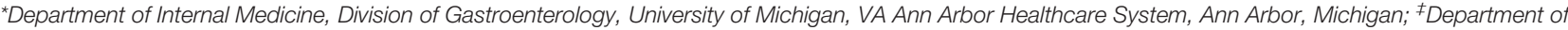 \\ Anesthesiology, University of Texas Medical Branch, Galveston, Texas; §Halberg Chronobiology Center, University of Minnesota, Minneapolis, Minnesota; \\ "Department of Internal Medicine, Division of Nephrology, University of Michigan, Ann Arbor, Michigan; "Department of Internal Medicine, Division of \\ Gastroenterology, University of Texas Medical Branch, Galveston, Texas; and "Department of Biology, Texas A\&M University, College Station, Texas
}

\section{See editorial on page 1373.}

Background \& Aims: Based on observations that the gastrointestinal tract is subject to various 24hour rhythmic processes, it is conceivable that some of these rhythms are under circadian clock gene control. We hypothesized that clock genes are present in the gastrointestinal tract and that they are part of a functional molecular clock that coordinates rhythmic physiologic functions. Methods: The effects of timed feeding and vagotomy on temporal clock gene expression (clock, bmal1, per1-3, cry1-2) in the gastrointestinal tract and suprachiasmatic nucleus (bmal, per 2 ) of C57BL/6J mice were examined using real-time polymerase chain reaction and Western blotting (BMAL, PER2). Colonic clock gene localization was examined using immunohistochemistry (BMAL, PER1-2). Results: Clock immunoreactivity was observed in the myenteric plexus and epithelial crypt cells. Clock genes were expressed rhythmically throughout the gastrointestinal tract. Timed feeding shifted clock gene expression at the RNA and protein level but did not shift clock gene expression in the central clock. Vagotomy did not alter gastric clock gene expression compared with shamtreated controls. Conclusions: The murine gastrointestinal tract contains functional clock genes, which are molecular core components of the circadian clock. Daytime feeding in nocturnal rodents is a strong synchronizer of gastrointestinal clock genes. This synchronization occurs independently of the central clock. Gastric clock gene expression is not mediated through the vagal nerve. The presence of clock genes in the myenteric plexus and epithelial cells suggests a role for clock genes in circadian coordination of gastrointestinal functions such as motility, cell proliferation, and migration.

$\mathrm{D}$ aily biologic rhythms and the clocks that coordinate them are fundamental properties of most organisms. ${ }^{1}$ Processes ranging from transcription to phys- iology and behavior are expressed predominantly at one time of day or another, synchronizing their peak activity with external time cues. These daily biologic rhythms are regulated by endogenous oscillators that express rhythmic function with a period close to 24 hours (circadian). Circadian oscillators are in turn synchronized or entrained on a daily basis to environmental cues so as to maintain a stable phase relationship with the 24-hour time span of the day.

Various gastrointestinal functions show a daily rhythmicity. ${ }^{2-4}$ Gastric acid secretion increases at night, and colonic motor activity increases in the morning..$^{5,6}$ Daily variations in expression of individual proteins such as the intestinal $\mathrm{Na}^{+}$/glucose cotransporter and Toll-like receptors have been described as well.7,8 Disruption of daily rhythms, which occurs with shift work or transmeridian traveling, has been associated with gastrointestinal symptoms such as abdominal bloating, abdominal pain, diarrhea, or constipation. ${ }^{9}, 10$ These observations strongly suggest a functional correlation between biologic rhythms and gastrointestinal physiology.

The hypothalamic suprachiasmatic nucleus (SCN) influences the expression of all circadian rhythms in mammals. ${ }^{11}$ Ablation of the SCN abolishes or dampens circadian rhythms in locomotor activity, sleep-wake cycles, body temperature, heart rate, and feeding. Circadian functions of feeding may depend on a separate foodentrainable oscillator. ${ }^{12}$ Even though an SCN lesion abolishes patterns of ad libitum feeding, SCN-lesioned rats and mice will entrain to a scheduled feeding regimen. An anticipatory feeding rhythm persists when the rodents are transferred to ad libitum food following the restricted feeding protocol. These data suggested early on that food-associated oscillation is influenced rather than driven by the SCN pacemaker.

Molecular mechanisms underlying circadian rhythms are conceived as a series of interlocking molecular loops 
involving rhythmic transcription of specific "clock genes" and the interactions of the proteins they encode. These clock genes comprise "positive elements" clock and bmal1, whose protein products dimerize, enter the nucleus, and stimulate transcription of "negative elements" period 1, 2, and 3 (per1-3) and cryptochrome 1 and 2 (cry1-2). The protein products of these genes (PER1-3, CRY1-2) in turn oligomerize, enter the nucleus, and suppress the activity of the CLOCK/BMAL1 complex. ${ }^{1,13}$ Other members of this set of interacting genes are reviewed elsewhere. ${ }^{1}$

Clock genes are also expressed rhythmically in peripheral organs in which they can presumably coordinate expression of a subset of tissue-specific genes, which may in turn impact directly on their physiologic functions. ${ }^{13}$ Although the central clock is light responsive and can be synchronized or reset by environmental cues such as the light/dark cycle, peripheral clocks cannot perceive light. These may be entrained by the central clock or independently by other physiologic stimuli such as feeding. The mechanism of interaction through which the central clock entrains peripheral clocks is unknown. ${ }^{13}$

Based on observations that the gastrointestinal tract is subject to various 24 -hour rhythmic processes, it is conceivable that some of these rhythms are under circadian clock gene control, whereas others may turn out to be dependent on other factors. We hypothesized that clock genes are present in the gastrointestinal tract and that they are part of a functional molecular clock that coordinates rhythmic physiologic functions.

\section{Materials and Methods}

\section{Animals}

Male C57BL/6J mice (8-12 weeks of age) were purchased from Jackson Laboratory (Bar Harbor, ME). Experimental protocols were approved by the Institutional Animal Care and Use Committee (IACUC) in accordance with the guidelines provided by the National Institutes of Health.

\section{Circadian Experiments}

Mice were maintained for 2 weeks on a light/dark cycle of 12 hours light:12 hours darkness (lights on 0700, lights off 1900; light/dark cycle 12:12) prior to all experiments. For the reference experiment, mice were kept in light/dark cycle with ad libitum access to food. Samples were collected every 4 hours starting 1 hour after the lights went on for 48 hours. To determine whether rhythms of clock gene expression persist in constant darkness, mice were kept in constant darkness for a total of 48 hours with ad libitum access to food. In this case, samples were collected in constant darkness at the same times as those collected in light/dark cycle. For timed feeding experiments, mice were kept for a total of 48 hours or 1 week on a regular light/dark cycle with access to food for 4 hours (0800-1200). For all experiments, animals were anesthetized with ketamine $(100 \mathrm{mg} / \mathrm{kg})$ and xylazine $(10 \mathrm{mg} / \mathrm{kg})$ prior to laparotomy. The gastric body was resected. Proximal colonic tissue was resected approximately $1.5 \mathrm{~cm}$ from the cecum, midcolon was resected approximately $3 \mathrm{~cm}$ from the cecum, and distal colon was resected approximately $0.5 \mathrm{~cm}$ above the anus. Mice were killed by decapitation. The brain was removed from the skull and frozen in powdered dry ice.

\section{Vagotomy}

Truncal vagotomy was performed by cutting the vagal trunks around the abdominal esophagus as previously described. ${ }^{14}$ In brief, following a midabdominal laparotomy, the distal esophagus was identified, and the posterior and anterior branches of the vagal nerve were transected. In sham-treated animals, the branches were manipulated only. Tissues were resected 10 days postsurgery. Stomachs of vagotomized mice were grossly enlarged at the time of death consistent with the effect of vagotomy.

\section{Materials}

Antibodies used for fluorescent immunohistochemistry are as follows: PER1 1:100 (Abcam, Cambridge, MA), PER2 1:100 (Alpha Diagnostic International, San Antonio, TX), $\beta$-tubulin 1:250 (Aves Labs, Inc, Tigard, OR), and Alexa-conjugated secondary antibodies 1:200 (Molecular Probes, Leiden, The Netherlands). Antibodies used for nonfluorescent immunohistochemistry and Western blotting are as follows: PER2 1:200 (Chemicon, Temecula, CA), Bmal 1:400 (Novus, Littleton, CO), and actin 1:1000 (Santa Cruz Biotechnology Inc, Santa Cruz, CA).

\section{Tissue Processing and Immunohistochemistry}

Frozen sections. Mice were transcardially perfused and fixed with $4 \%$ paraformaldehyde. Tissues were removed, postfixed in $4 \%$ paraformaldehyde ( 1 hour) at room temperature, and cryoprotected by infiltration in $30 \%$ sucrose/phosphate-buffered saline (PBS) overnight at $4^{\circ} \mathrm{C}$. The tissue was placed in Optimal Cutting Temperature embedding medium (Tissue Tek, Sakura, Tokyo) and frozen over dry ice. Frozen sections $(10 \mu \mathrm{m})$ were cut at $-15^{\circ} \mathrm{C}$ on a cryostat, placed on plus slides (VWR, West Chester, PA), and stored at $-80^{\circ} \mathrm{C}$.

Immunohistochemistry. Tissue was rehydrated in PBS (20 minutes) at room temperature, blocked with 5\% normal serum (1 hour), washed in PBS, and incubated with primary antibodies diluted in $1.5 \%$ normal serum overnight at $4^{\circ} \mathrm{C}$. Slides were rinsed in PBS, incubated with Alexa-conjugated secondary antibodies (1 hour) at room temperature, washed in PBS, and mounted with FluorSave (Calbiochem, Darmstadt, Germany). Controls for specificity of immunolabeling included omission of the primary antibody from the immunostaining procedure. 
Table 1. Clock Gene Primers for Conventional PCR

\begin{tabular}{|c|c|c|}
\hline Primers & Sequence & $\begin{array}{c}\text { Accession } \\
\text { number }\end{array}$ \\
\hline CLOCK-FW & CCAGCACATGATACAGCAAC & AF000998 \\
\hline CLOCK-RV & GAAGGAAGCTGCTGTTCCTG & \\
\hline BMAL1-FW & CTATCTTCCTCGGACACTGC & ВC025973 \\
\hline BMAL1-RV & СTTCTTGCСTCCTGGAGAAG & \\
\hline PER1-FW & GGAGCTACTGCTCCAAGAAG & AF022992 \\
\hline PER1-RV & CCATGGCAGAGTCCTGAGAG & \\
\hline PER2-FW & CTGCACATCTGGCACATCTC & AF035830 \\
\hline PER2-RV & GCTTCTGAGGTATCACAGAG & \\
\hline PER3-FW & CGTTCATTAGCTCACGGAGC & AF050182 \\
\hline PER3-RV & CTGTGTGGCTGTGGATCCAG & \\
\hline CRY1-FW & GCTTGCAAGCCCAACTGGAC & ВC085499 \\
\hline CRY2-RV & GTCTCCATTGGGATCTGTCC & \\
\hline CRY2-FW & GTTTGGCAAGGAGGAGAGAC & ВC054794 \\
\hline CRY2-RV & GTCTCCATTGGGATCTGTCC & \\
\hline
\end{tabular}

FW, forward; RV, reverse.

\section{Imaging}

Images were captured using an Olympus BX60 fluorescent microscope (Olympus, Melville, NY) equipped with Metaview software (Universal Imaging Corp, West Chester, PA).

\section{Total RNA Extraction}

Total RNA was isolated using TRIzol Reagent (Invitrogen, Carlsbad, CA) and treated with Dnase I (Promega, Madison, WI) at $37^{\circ} \mathrm{C}$ for 30 minutes.

\section{Reverse-Transcription Polymerase Chain Reaction}

Reverse-transcription polymerase chain reaction (RT-PCR) (ProSTAR Ultra HF RT-PCR System; Strat- agene, La Jolla, CA) was performed using clock genespecific primers (Table 1: nucleotide sequence) as follows: initial denaturation $95^{\circ} \mathrm{C}(1$ minute $), 95^{\circ} \mathrm{C}(1$ minute), $56^{\circ} \mathrm{C}$ (1 minute), $72^{\circ} \mathrm{C}$ (1 minute) for 35 cycles, $72^{\circ} \mathrm{C}$ (10 minutes) final extension. Fragments were ligated into pGEM 5Z $(\mathrm{f})+$ (Promega) and sequenced.

\section{Real-time PCR}

TAQMAN 2-step real-time PCR was performed using reagents from Applied Biosystems (Foster City, CA) on a Chromo4 Continuous Fluorescence Detector (Bio-Rad, Hercules, CA). Primers were designed with Primer Express software from ABI (Applied Biosystems), and fluorescent probes were ordered from Integrated DNA Technologies, Inc (Coralville, IA). (Table 2: primer-probe sequences). To develop a real-time multiplex PCR assay, probes for individual genes were labeled with reporter dyes (fluorophores) of different wavelengths (FAM, Texas-Red, and Cy5). Probes were initially used in single PCR amplification reactions to establish maximum amplification efficiency. Subsequently, primers and probes for 2 individual genes were combined with the $18 \mathrm{~S}$ control (VIC) and used on samples in 1 single PCR reaction. Thermal cycling conditions were as follows: $50^{\circ} \mathrm{C}(2$ minutes $), 95^{\circ} \mathrm{C}(10$ minutes), $95^{\circ} \mathrm{C}$ (15 seconds), and $60^{\circ} \mathrm{C}(1$ minute) for 40 cycles. Real-time PCR for vagotomized and shamoperated mice were completed with TaqMan Gene Expression Assays from Applied Biosystems containing primer/probe sets for clock, bmal, per1-3, and cry1 on an Eppendorf MCEP Realplex (Westbury, NY).

Table 2. Clock Gene Primers and Probes for Real-time PCR

\begin{tabular}{|c|c|c|c|c|}
\hline Primers & $5^{\prime}$ & $3^{\prime}$ & Sequence & Accession number \\
\hline CLOCK-FW & & & TCTACAGAAGAGCATTGATTTTTTGC & AF000998 \\
\hline CLOCK-RV & & & TCATTACTAAGGAATGTGGGTTTCC & AF000998 \\
\hline CLOCK-Probe & DFAM & DTAM & TGCACAGTCAGATGCTAGTGAGATTCGACA & AF000998 \\
\hline BMAL-FW & & & CGTTTCTCGACACGCAATAGAT & AB014494 \\
\hline BMAL-RV & & & TCCTGTGGTAGATACGCCAAAA & AB014494 \\
\hline \multirow[t]{2}{*}{ BMAL-Probe } & DFAM & DTAM & AATTTGTTTTTGTAGATCAGAGGGCGACAGCTAG & AB014494 \\
\hline & & & CTGTCGCCCTCTGATCTACAAAAACAAATT & \\
\hline PER1-FW & & & CACTGAGAGCAGCAAGAGTACAAACT & AF022992 \\
\hline PER1-RV & & & AGATGGGTTGTCCTGCTCTGA & AF022992 \\
\hline PER1-Probe & DTXR & $\mathrm{DBH} 2$ & AGCAGCTCCATTGCCTACAGCCTCCT & AF022992 \\
\hline PER2-FW & & & GATGACAGAGGCAGAGCACAAC & AF035830 \\
\hline PER2-RV & & & TTTGTGTGCGTCAGCTTTGG & AF035830 \\
\hline PER2-Probe & DCY5 & $\mathrm{DBH} 2$ & CCTCCACGAGCGGCTGCAGTAGTG & AF035830 \\
\hline PER3-FW & & & GGTCCTCTGCAGGGCACTT & AF050182 \\
\hline PER3-RV & & & TTCAGACATTCTGTTTCGGTCTTC & AF050182 \\
\hline PER3-Probe & DFAM & DTAM & CGTGGACAGCAGCCACAGTGAACA & AF050182 \\
\hline CRY1-FW & & & CCCTGTGGGTTTTGGTAGGA & AB000777 \\
\hline CRY1-RV & & & TGCAGGGAAGCCTCTTAGGA & AB000777 \\
\hline CRY1-Probe & DFAM & DTAM & CAGATCCCAATGGAGACTATATTAGGCGTTATTTACC & AB000777 \\
\hline CRY2-FW & & & GGCGTGGAGGTGGTGACT & AB003433 \\
\hline CRY2-RV & & & TGGTTTCTGCCCATTCAGTTC & AB003433 \\
\hline CRY2-Probe & DTXR & $\mathrm{DBH} 2$ & AGAACTCTCACACCCTCTATGACCTAGACAGAATCA & AB003433 \\
\hline
\end{tabular}

FW, forward; RV, reverse. 


\section{Laser Capture Microdissection}

Preparation of sections. Frozen sections $(10 \mu \mathrm{m})$ were cut on a cryostat, mounted on super-frost slides, and stored at $-20^{\circ} \mathrm{C}$. Slides were thawed at room temperature ( 30 seconds), fixed ( 1 minute) with $75 \%$ ethanol, and prepared for laser capture microdissection (LCM) as previously described. ${ }^{15}$

LCM. LCM was performed using a PixCell II laser capture microscope with an infrared diode laser (MDS
Analytical Technologies, Inc, Toronto Canada). Several thousand neurons from 6 to 8 sections of SCN were captured. The smallest laser spot size $(7.5 \mu \mathrm{m})$ was used with a power setting of $75-100 \mathrm{~mW}$ and pulse duration of $0.85-1.5 \mathrm{~ms}$. Both lobes of the SCN from 6-8 slides of each mouse brain were captured.

RNA isolation. Total RNA was extracted using the RNAqueous-Micro kit (Ambion, Austin, TX) according to modified manufacturer's protocol. DNase treat-
Figure 1. (A) Agarose gel showing RT-PCR products for parts of individual clock gene coding regions. Left lane shows the marker. No-RT controls are flanking clock gene amplimers to the right. Predicted amplimers sizes: clock, 491 bp; per1, 200 bp; per2, 991 bp; per3, 527 bp; cry1, 551 bp; cry2, 600 bp; bmal1, 992 bp. (B) Images of distal colon with hematoxylin counter staining (original magnification, $\times 40$ ). Thin black arrows point toward areas of positive (brown) staining cells in the myenteric plexus, and thick black arrows point toward areas of positive staining of epithelial cells for PER2 antibody and BMAL antibody. (C) Images of double labeling immunohistochemical staining of PER1 and PER2 (green) with $\beta$-tubulin (red) in the murine colon. The overlap image represents superimposition of fluorescent and bright field images. $L M$, longitudinal muscle; $C M$, circular muscle, $M P$, myenteric plexus. Bar, $20 \mu \mathrm{m}$. Arrows point toward area of overlapping staining in the myenteric plexus.

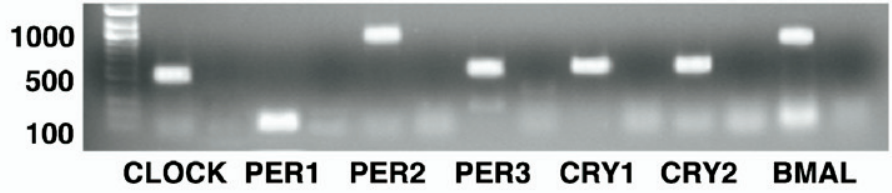

Per2
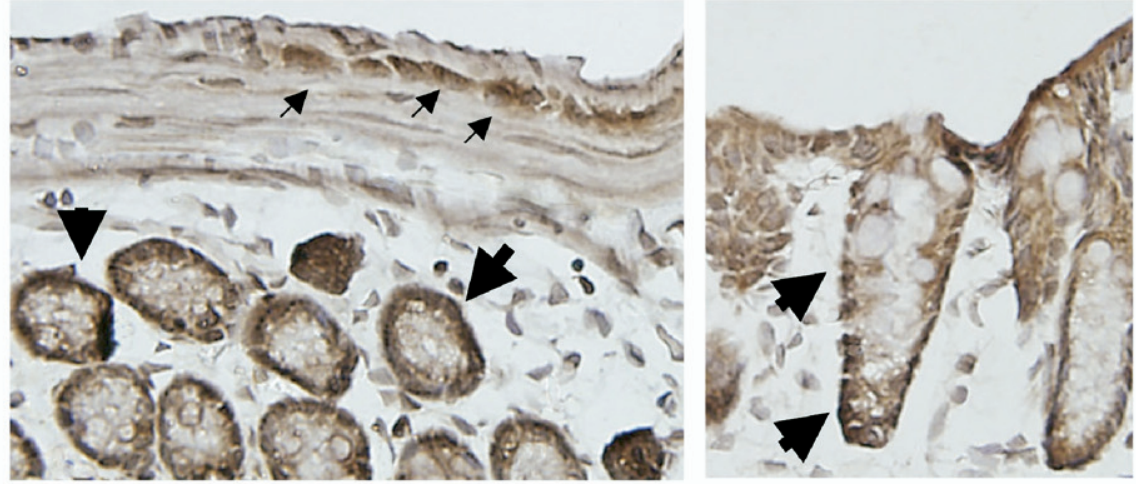

Bmal
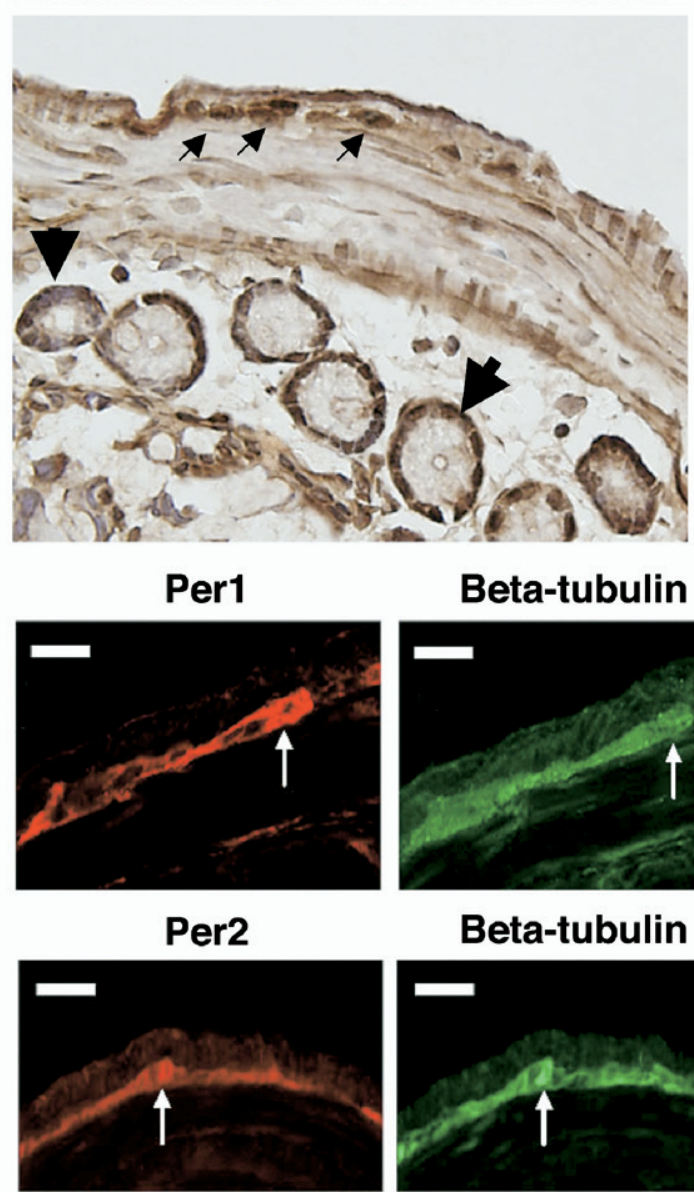

Beta-tubulin

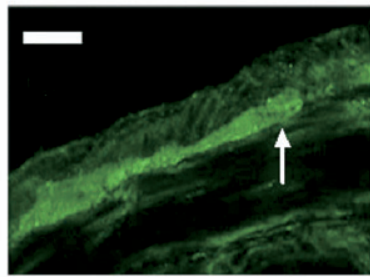

Beta-tubulin

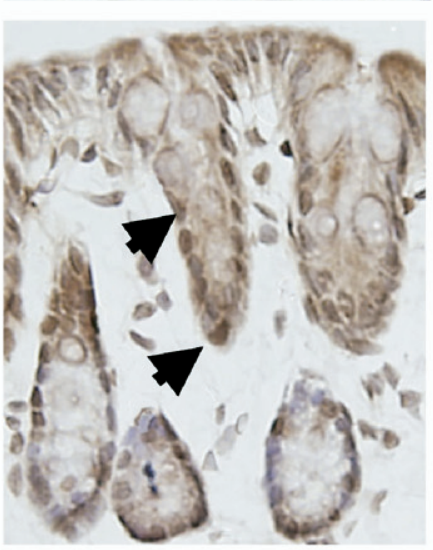

Overlap

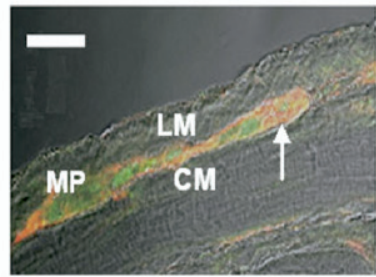

Overlap
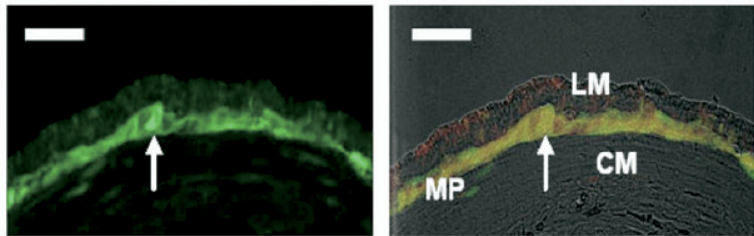
ment was performed to remove genomic DNA. DNase was subsequently removed with DNase Inactivation Reagent.

\section{Western Blotting}

Tissue was homogenized in buffer with $2 \%$ sodium dodecyl sulfate, $100 \mu \mathrm{mol} / \mathrm{L}$ protease cocktail inhibitor, $1 \mathrm{mmol} / \mathrm{L}$ phenylmethylsulphonyl fluoride, 1 $\mathrm{mmol} / \mathrm{L}$ EDTA in $50 \mathrm{mmol} / \mathrm{L}$ Tris-buffered saline. Samples were centrifuged at $14,000 \mathrm{~g}$ (15 minutes) and supernatants transferred. Protein concentrations were determined by BCA protein assay (Pierce, Rockford, IL). Samples were diluted in sample buffer, boiled for 5 minutes, and loaded onto a $4 \%-20 \%$ Tris- $\mathrm{HCl}$ gel. After electrophoresis, proteins were electrotransferred onto nitrocellulose membrane and incubated in 5\% milk in Trisbuffered saline $/ 0.1 \%$ Tween 20 ( 1 hour) and primary antibodies overnight at $4{ }^{\circ} \mathrm{C}$ followed by horseradishperoxidase (HRP)-conjugated anti-rabbit Ig (1:5000 dilution) for 1 hour. Immunoreactive bands were detected by a chemiluminescent Western blot detection kit (Amersham Biosciences, Buckinghamshire, United Kingdom). Protein quantification was completed via computer image analysis (Scion Image; Scion Corp, Frederic, MD) using band integrated density. Data were normalized to actin.

\section{Statistical Analysis}

Gene expression was analyzed using the relative PCR amplification analysis method $\left(2^{-\Delta \Delta C t}\right)$. Changes in clock gene expression were referenced to the level of expression at the 0800 time point. Data are presented as means $\pm \mathrm{SE}(\mathrm{n}=3$ mice per time point). Cosinor analysis was then performed using fold increase over the 0800 time point to test for the presence of a circadian rhythm. Cosinor analysis adjusts data to a cosinusoidal function and provides an objective test of whether the amplitude of the cosinor curve differs from zero, ie, whether a rhythm is validated for the assumed period ( 24 hours herein). This method provides point and confidence interval estimates for 3 parameters describing the cosine curve: the MESOR (M, midline estimating statistic of rhythm, a rhythm-adjusted mean), the double amplitude $(2 \mathrm{~A}$, a measure of the extent of predictable change within 1 cycle), and the acrophase (a measure of the timing of the peak value recurring in each cycle). These parameters are adjusted by least squares, a method also yielding a coefficient of determination, $R^{2}$, known as the percentage
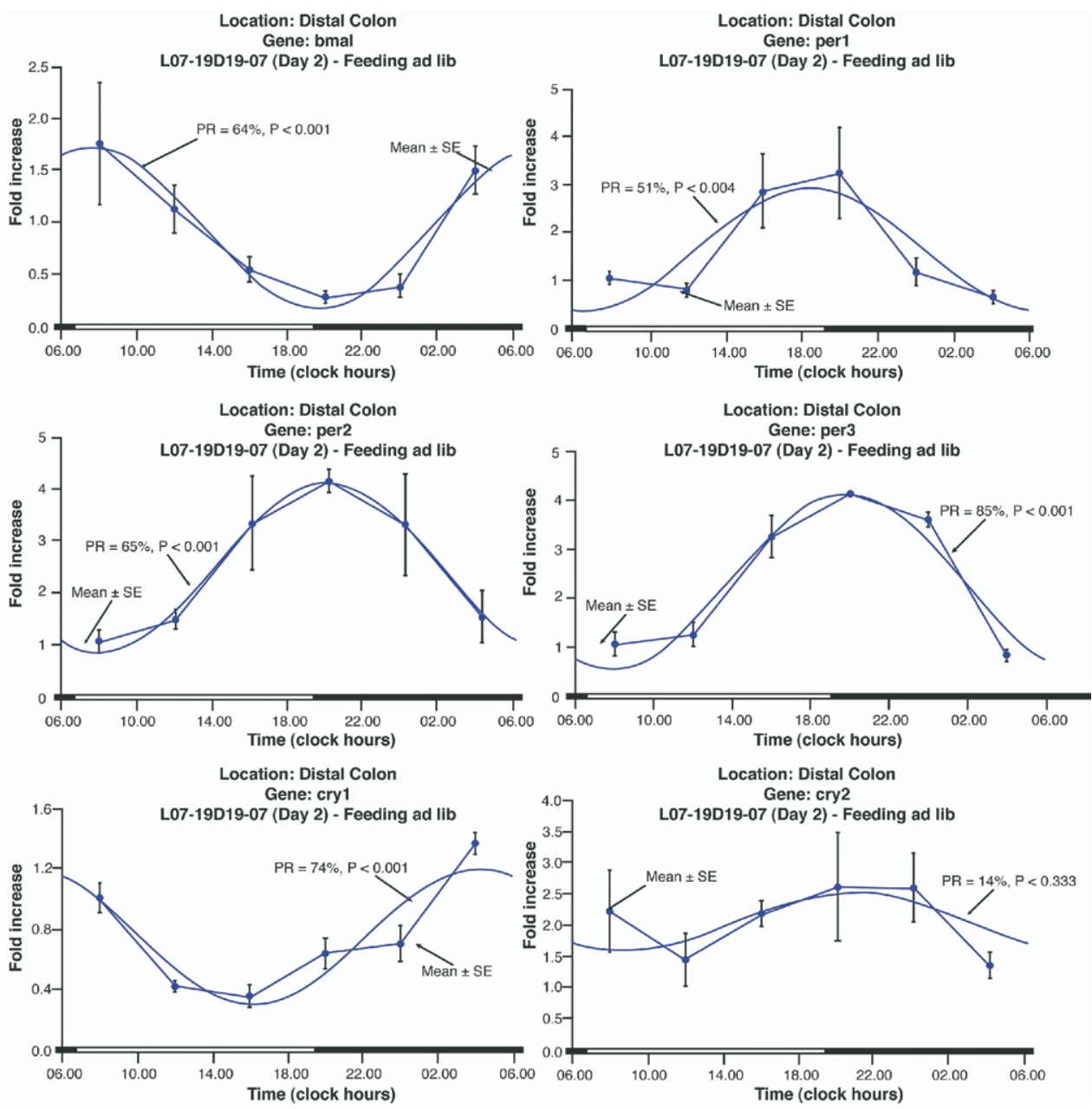

Figure 2. Daily profiles of colonic clock gene mRNA levels and corresponding fitted cosinor curves. The mRNA levels are expressed as means $\pm \mathrm{SE}$ ( $\mathrm{n}=3$ mice per time point). $P R$, percentage rhythm; $P$ value, probability from zero-amplitude (no rhythm) test. 
rhythm (PR), representing the proportion of the total variance accounted for by the fitted model. An F test of the null hypothesis $\mathrm{H}_{0}: \mathrm{A}=0$ determines the statistical significance of the model. To determine whether different experimental conditions affect circadian acrophase and amplitude for a given clock gene, real-time PCR data had to be expressed in relative values because the 0800 value was not calibrated. For each data series (18 values, 3 at each of 6 time points, 4 hours apart), the arithmetic mean was calculated, and the data were expressed as a percentage of that mean value. In this way, the average was $100 \%$ in all cases, and comparisons could be made of relative amplitudes and of acrophases. Parameter tests were used to test the equality of relative circadian amplitudes and of circadian acrophases among 2 or more data series. ${ }^{16}$ Because the acrophase is a circular variable, differences in acrophase exceeding $90^{\circ}$ cannot be tested. ${ }^{16}$ Hence, statistically significant differences in acrophase were also sought by determining whether clock genes had acrophases with nonoverlapping 95\% confidence intervals. Significance levels were set at $P<.05$.

\section{Results}

\section{Clock Genes Are Expressed in the Murine Stomach and Colon}

Parts of the coding region of the clock genes were amplified from adult mouse stomach and proximal, middle, and distal colon by RT-PCR from total RNA (Figure $1 A$; data shown for proximal colon only). PCR products were all TA cloned, and DNA sequence analysis confirmed the identity of all clock genes.

PER1, PER2, and BMAL protein expression in the gastrointestinal tract were confirmed by immunohistochemistry in tissue sections obtained at the 0800 and 2000 time points, with no difference in staining intensity between the 2 time points. No immunostaining was identified with secondary antibodies only (data not shown). Prominent PER2 and BMAL immunoreactivity were noted in epithelial cells along the colonic crypts with most intense staining in the bases of the crypts (Figure $1 B$ ). Prominent PER2 and BMAL immunoreactivity were detected in the myenteric plexus of the stomach and

Table 3. Circadian Rhythm Characteristics in the Murine Stomach and Colon

\begin{tabular}{|c|c|c|c|c|c|}
\hline Gene & MESOR, M & Amplitude, A & Acrophase, $\Phi(\mathrm{hh}: \mathrm{mm})$ & Percentage rhythm (\%) & $P$ value $\left(\mathrm{H}_{0}: \mathrm{A}=0\right)$ \\
\hline \multicolumn{6}{|c|}{ Stomach } \\
\hline clock & 1.066 & 0.364 & 09:52 & 17 & .243 \\
\hline bmal & 0.564 & 0.511 & $06: 28$ & 65 & .001 \\
\hline per1 & 0.741 & 0.150 & $02: 44$ & 5 & .672 \\
\hline per2 & 5.128 & 5.162 & $21: 16$ & 79 & $<.001$ \\
\hline per3 & 1.048 & 0.473 & 19:04 & 43 & .015 \\
\hline cry1 & 0.835 & 0.230 & $02: 48$ & 28 & .117 \\
\hline cry2 & 1.242 & 0.368 & $22: 24$ & 32 & .058 \\
\hline \multicolumn{6}{|c|}{ Proximal colon } \\
\hline clock & 4.692 & 1.099 & $07: 56$ & 24 & .124 \\
\hline bmal & 0.813 & 0.805 & 05:56 & 82 & $<.001$ \\
\hline per1 & 3.418 & 2.259 & $17: 40$ & 57 & .002 \\
\hline per2 & 5.849 & 3.833 & 01:40 & 59 & .001 \\
\hline per3 & 9.698 & 7.380 & $17: 52$ & 83 & $<.001$ \\
\hline cry1 & 1.411 & 0.549 & $02: 28$ & 41 & .020 \\
\hline cry2 & 3.313 & 0.999 & $20: 40$ & 27 & .098 \\
\hline \multicolumn{6}{|c|}{ Mid colon } \\
\hline clock & 1.773 & 0.652 & $08: 36$ & 23 & .137 \\
\hline bmal & 0.828 & 0.736 & 06:08 & 64 & $<.001$ \\
\hline per1 & 0.473 & 0.289 & 18:08 & 79 & $<.001$ \\
\hline per2 & 3.007 & 2.135 & $22: 12$ & 71 & $<.001$ \\
\hline per3 & 0.941 & 0.770 & $18: 28$ & 92 & $<.001$ \\
\hline cry 1 & 1.077 & 0.510 & $03: 52$ & 49 & .006 \\
\hline cry2 & 3.678 & 1.322 & $20: 24$ & 54 & .003 \\
\hline \multicolumn{6}{|c|}{ Distal colon } \\
\hline clock & 0.934 & 0.153 & $17: 56$ & 5 & .674 \\
\hline bmal & 0.168 & 0.050 & $04: 40$ & 17 & .251 \\
\hline per1 & 1.703 & 0.458 & 19:32 & 23 & .148 \\
\hline per2 & 2.694 & 2.557 & $21: 12$ & 84 & $<.001$ \\
\hline per3 & 1.842 & 1.103 & $19: 16$ & 58 & .001 \\
\hline cry 1 & 1.681 & 1.004 & 01:04 & 68 & $<.001$ \\
\hline cry2 & 0.833 & 0.333 & 21:04 & 58 & .002 \\
\hline
\end{tabular}

NOTE. MESOR (M) refers to midline estimating statistic of rhythm, a rhythm-adjusted mean; amplitude refers to a measure of the extent of predictable change within 1 cycle; and the acrophase refers to a measure of the timing of overall high values recurring in each cycle. The $P$ value refers to the probability from the zero-amplitude (no rhythm) test.

hh:mm; hour:minutes. 
throughout the colon. Protein expression of PER1 and PER2 in the myenteric plexus was further supported by its colocalization with $\beta$-tubulin, a neuronal marker (Figure $1 C)$.

\section{Clock Genes Are Expressed in a Circadian Manner in the Murine Stomach and Colon}

Real-time PCR was used to assess whether intestinal clock genes are expressed in a rhythmic manner under ad libitum feeding conditions in a regular light/ dark cycle. Rhythmic expression patterns were observed for 2 consecutive days for all clock genes in the stomach and throughout the colon (Figure 2: data shown for distal colon, day 2). There was no statistically significant difference in the circadian amplitude or acrophase of individual clock genes in the stomach or the colon on day 1 vs day 2 , in keeping with the presence of a 24-hour cycle.

To determine whether rhythmically expressed clock genes in the gastrointestinal tract were driven by the light-dark cycle, mice were housed in constant darkness to eliminate the effect of light as a synchronizer of clock gene expression, with ad libitum access to food. Table 3 summarizes the circadian rhythm characteristics for the individual clock genes in the stomach and colon. Under dark/dark conditions, clock genes largely maintained rhythmic expression in the stomach and throughout most of the colon except for clock. Under dark/dark conditions but with ad libitum access to feeding, the circadian amplitude was decreased for most clock genes throughout the gastrointestinal tract. This difference was only statistically significant in the proximal colon.

We examined whether differences in acrophase and amplitude existed among the 4 different sites and between adjacent sites (stomach vs proximal, middle, and distal colon; proximal vs middle; and middle vs distal colon) for individual clock genes. The phases of all examined clock gene mRNA profiles were synchronous throughout the colon. When aligning the rhythmically expressed clock genes in order of their acrophase, cry 1 and Bmal1 peak before other clock genes, followed by per1-3 and cry2 (Figure 3; data shown for distal colon only) in stomach, proximal, middle, and distal colon. This order remained largely unchanged under constant darkness.

\section{Colonic Clock Gene Expression Shifts in Response to Timed Feeding}

To examine whether feeding time can affect the phase of colonic clock gene expression, mice were fed for 48 hours or for 1 week during the day from 0800 until 1200. Following the restricted feeding time span, the phase of different clock genes was determined. The phases of all clock gene mRNA accumulation profiles in the stomach and throughout the colon differed 7 to 12

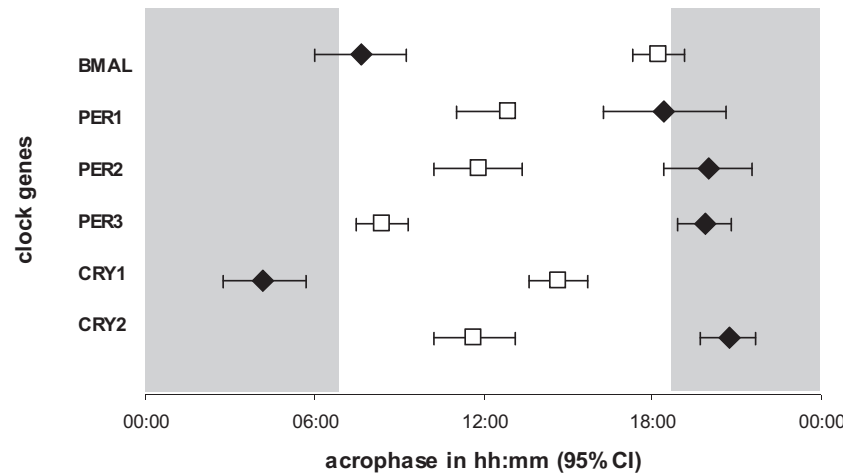

Figure 3. Acrophase of distal colonic clock genes for mice with ad libitum food access (solid squares) and following 48 hours of timed feeding (open squares). Bars represent 95\% confidence intervals. Grey shade indicates dark cycle.

hours between mice fed during the daytime and mice fed ad lib (Figure 3; data shown for distal colon). Because the degree of the phase shift differed between individual clock genes, we examined whether prolonged food restriction would further phase shift clock gene expression. Following 1 week of restricted feeding, no additional phase shifting occurred (data not shown).

\section{Colonic Clock Gene Protein Expression Is Expressed in a Circadian Manner and Shifts in Response to Timed Feeding}

To examine whether clock genes are expressed in a circadian manner at the protein level, intestinal tissues were collected following ad libitum feeding and 48 hours of timed feeding. Data were obtained for BMAL, a "positive element" of the feedback loop, and for PER2, a "negative element" of the feedback loop (Figure 4). No bands were identified with a secondary antibody only control (data not shown). The acrophase for BMAL was at 13:40 (95\% CI: 10:48-16:32 in hh:mm) under ad libitum feeding and at 21:02 (95\% CI: 19:04-23:08 in hh: $\mathrm{mm})$ following timed feeding. The acrophase for PER2 was at 00:00 (95\% CI: 22:16-01:40 in hh:mm) under ad libitum feeding and at 11:56 (95\% CI: 19:04-23:08 in hh:mm) following timed feeding.

\section{Vagotomy Does Not Alter Circadian Clock Gene Expression}

To determine the role of vagal neurotransmission in gastric circadian clock gene expression, expression patterns were examined in vagotomized and shamtreated mice. Circadian rhythm was detected with statistical significance for the clock genes bmal, per1-3, and cry 1 in both vagotomized and sham-treated controls (data not shown). Circadian characteristics such as acrophase and amplitude were also not statistically signif- 
A

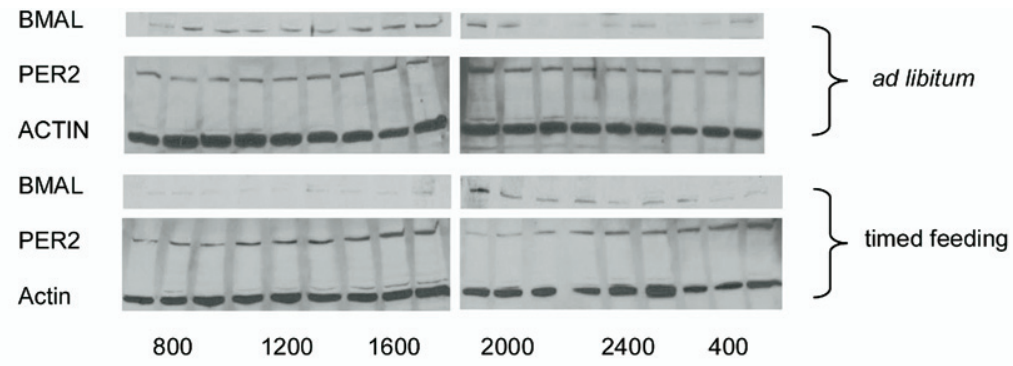

B
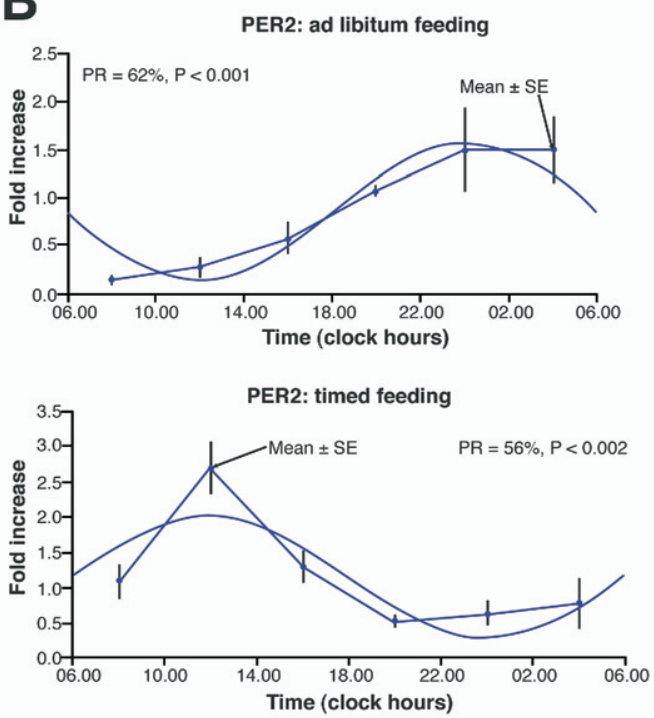
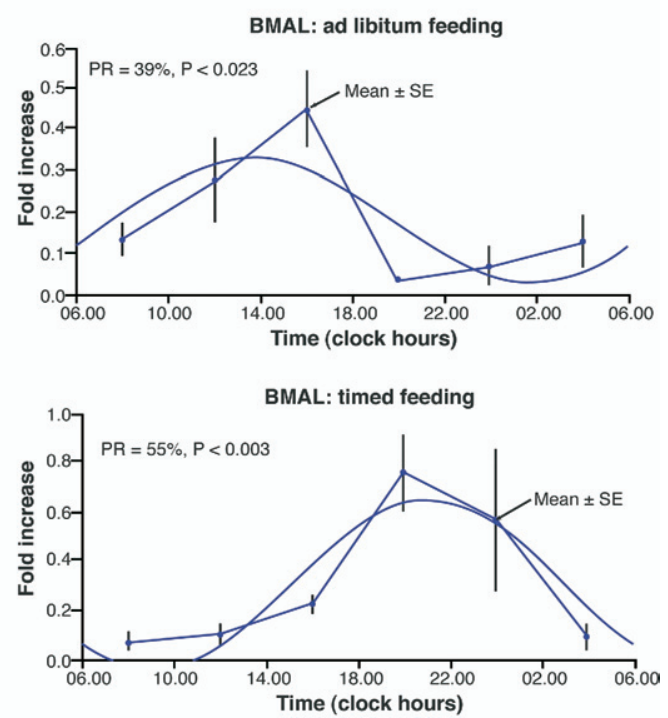

Figure 4. (A) Western blotting for clock proteins (BMAL and PER2) in mice ( $\mathrm{n}=3$ mice per time point) with ad libitum access to food and following 48 hours of timed feeding. (B) Quantitative analysis of clock gene protein rhythms over a 24-hour time span and corresponding fitted cosinor curves.

icantly different between vagotomized and sham-treated controls.

\section{Timed Feeding Does Not Shift Central Clock Gene Expression}

To examine whether timed feeding can affect the phase of central clock gene expression, the mice brains were collected following ad libitum feeding, timed feeding for 48 hours, and timed feeding for 1 week. Data were obtained for bmal and per2. Rhythmic expression patterns were detected with borderline statistical significance for per2 $(P=.093)$ and with statistical significance for bmal $(P=.036)$ under ad libitum feeding conditions. Per2 reached a near statistically significant rhythm $(P=.05)$ following 1 week of timed feeding but not at 48 hours, whereas $b m a l$ reached borderline statistical significance $(P=$ .074) following 48 hours of timed feeding but not at 1 week.

The acrophase for mice with ad libitum access to food was 16:12 and 04:40 for per2 and bmal, respectively. Following timed feeding for 48 hours and 1 week, the per 2 acrophase was 14:24 and 15:08, respectively; the bmal acrophase was 03:12 and 02:04, respectively. No statistically significant difference in either acrophase or amplitude was found among the 3 different conditions for either per2 or bmal (Figure 5).

\section{Discussion}

This study shows that a set of known mammalian clock genes, whose coordinated function is thought to regulate circadian rhythms, persist in their rhythmicity in constant darkness and can be synchronized in their expression patterns to changes in feeding schedule within 48 hours in the murine stomach and proximal, middle, and distal colon. This change in expression pattern occurs independently of the central clock. In addition, rhythmic gastric clock gene expression is not mediated by the vagal nerve.

Although previous studies have described the presence of a subset of clock genes in the murine and rat intestine, no study has systematically determined the presence, localization, and temporal expression of the main components of the mammalian clock in the murine gastrointestinal tract. ${ }^{17,18}$ PER1, PER2, and BMAL protein expression were identified in the colonic epithelial crypt cells and in the gastric and colonic myenteric plexus. The presence of clock genes in the myenteric plexus was supported by the colocalization of clock genes with $\beta$-tubulin, a neuronal marker.

Several studies have demonstrated that changes in feeding schedule can shift the acrophase of hepatic clock 

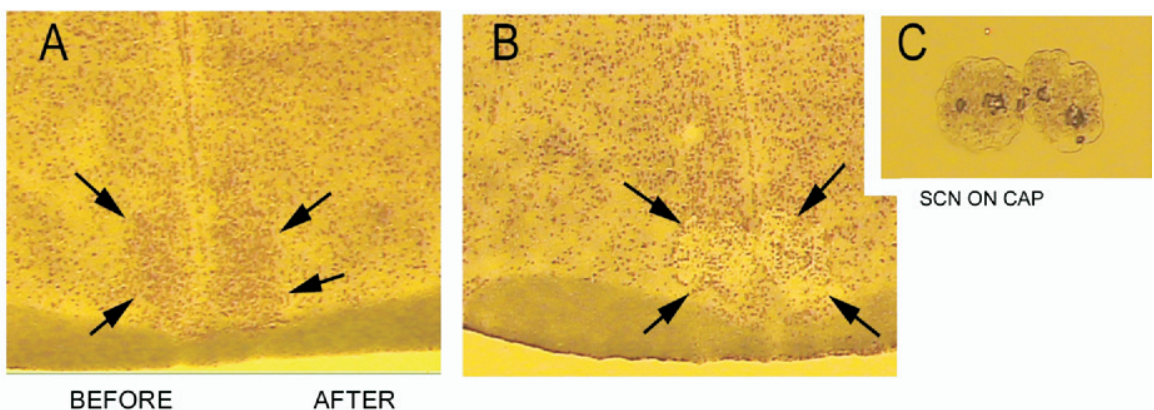

\section{D}

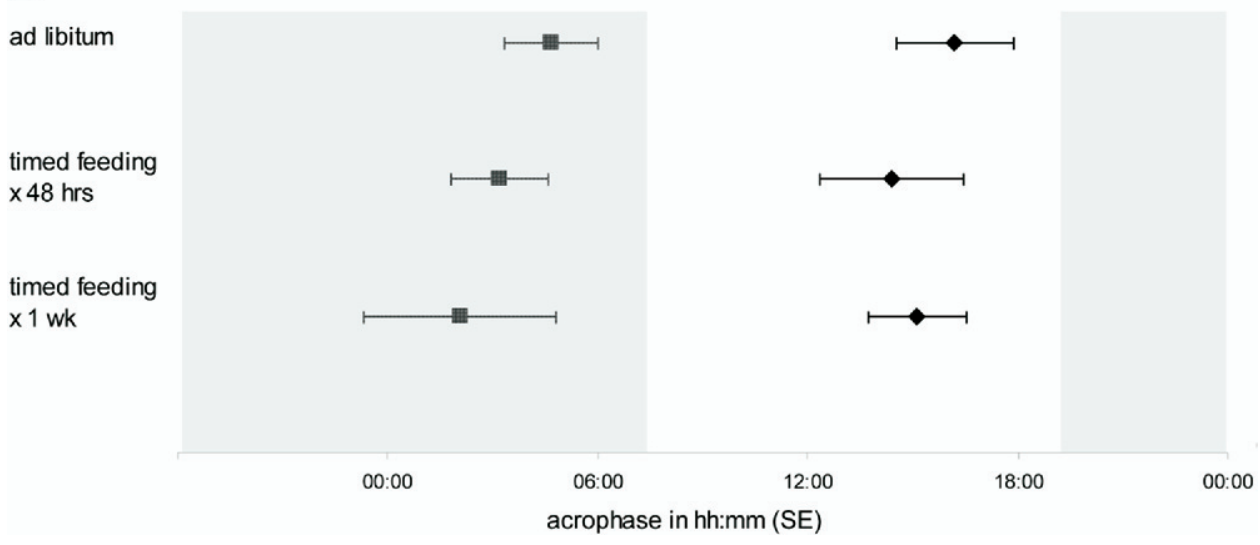

Figure 5. $(A-C)$ Cresyl violet stained coronal section of the mouse brain (original magnification, $\times 20$ ). Arrows delineate the SCN, flanking the third ventricle, before LCM $(A)$ and after LCM (B). Panel $C$ shows SCN on cap. (D) Acrophase for bmal and per2 in the SCN for mice with ad libitum access to food and following 48 hours or 1 week of timed feeding. Solid box represents bmal; diamond-shaped box represents per2. Bars represent SE. Grey shade indicates dark cycle.

genes ${ }^{19-21}$ but not pulmonary clock genes. ${ }^{21}$ Thus, it appears that clock gene expression in those organs that are intimately involved with feeding such as the gastrointestinal tract and the liver are most responsive to changes in feeding schedules. It has recently been demonstrated that clock genes can shift their expression patterns in response to timed feeding in extraintestinal tissues such as adipose tissue as well. ${ }^{22}$

The mechanism by which timed feeding alters the phase of peripheral clock gene expression remains poorly understood. ${ }^{20}$ Although timed feeding has been associated with an increase in food-anticipatory behavior (increased locomotor activity preceding a daily scheduled meal), food-anticipatory behavior by itself does not shift the phase of clock genes. ${ }^{18}$ It is also unlikely that signals directly associated with feeding such as the taste of food, stomach distention, or direct physical contact of food with the gastrointestinal lining play a significant role in clock gene expression. First, it has been shown that hepatic clock genes shifted their acrophase while receiving total parenteral nutrition during the light cycle, thus completely bypassing direct contact of food with the gastrointestinal epithelium. ${ }^{23}$ Second, we found no significant difference in acrophases of individual clock genes from the stomach to the distal colon. The lack of a significant difference in the phases of clock gene expression between these functionally different parts of the gastrointestinal system leaves open the question of whether clock genes are coordinated by a central neuro- nal pacemaker (ie, the SCN) or a systemic hormonal mechanism in the gastrointestinal system.

It is unlikely that the SCN directs the change in peripheral clock gene expression in response to timed feeding. In the present study, timed feeding did not alter clock gene expression in the central clock. These findings are in line with observations by others ${ }^{19-21,24-27}$ and support the hypothesis that a food-entrainable oscillator exists independent of the SCN. Recent reports suggest that this food-entrainable oscillator may be present in the dorsomedial hypothalamic nucleus, a region involved in food intake and satiety. ${ }^{28,29}$ Furthermore, SCN-lesioned rats and mice will continue to entrain to a scheduled feeding regimen.

The present study also aimed to determine the effect of the vagal nerve in the circadian expression of gastric clock genes. The rationale for this experiment was 2-fold. First, it has been suggested that the communication of the SCN with peripheral organs such as the lung, the small intestine, and the pancreas may be mediated through the vagal nerve. ${ }^{30-32}$ Second, gastric emptying and gastric acid secretion vary with the time of the day. ${ }^{5,33}$ Both gastric motility and acid secretions are mediated by the vagal nerve. However, we found no difference in circadian expression of gastric clock genes in vagotomized mice as compared with sham-treated controls.

This study is limited by the fact that both RNA and protein studies were completed on whole gut wall tissues. 
Therefore, we cannot determine the relative contribution of the different cell types to the measured rhythmic expression of clock genes. The predominance of immunohistochemical staining in neurons and epithelial cells suggests that these are the likely sources of the noted rhythmic clock gene expression. Furthermore, the presence of a distinct single peak in oscillation for all clock genes suggests that the clock genes in the different cell types of the gut are in phase with each other.

What are the potential functional implications for gastrointestinal clock genes? Because gene array studies have shown that approximately $8 \%-10 \%$ of all genes in peripheral organs such as the heart and the liver are under circadian coordination, it is conceivable that a subset of gastrointestinal genes are under clock gene control as well. ${ }^{34}$ For example, clock gene expression was detected in the epithelial cells of the colonic crypts. Circadian variations in the mitotic index and DNA synthesis have been well described in a variety of tissues including the intestinal epithelium. 4,35,36 It has also been shown that the circadian clockwork can directly control cell division cycles in proliferating cells. ${ }^{37}$ The potential importance of rhythmicity in gastrointestinal epithelial cell proliferation is demonstrated by the following clinical observations. The efficacy of chemotherapy in the treatment of metastatic colorectal cancer as well as the development of chemotherapy-related complications such as mucosal toxicity vary with the time of administration. ${ }^{38}$ Along similar lines, the timing of radiation therapy may impact on the efficacy, incidence, and severity of radiation therapy-related complications. ${ }^{39}$ Finally, data from the Nurses Health Study have shown that the risk of colorectal cancer increases in nurses that participated in a rotating shift at least 3 nights per month for 15 or more years. ${ }^{40}$ Taken together, these observations suggest an important role for clock genes in the regulation of normal physiologic cell migration and proliferation as well as a potential role in the development of colon cancer.

In our study, clock gene expression was also detected in the myenteric plexus. The myenteric plexus is an important site of neurotransmitter synthesis and integrates gastrointestinal motility into recognizable patterns. ${ }^{41}$ Neurotransmitters expressed in the myenteric plexus such as vasoactive intestinal peptide have been identified in the neurons of the central clock of the brain as well, where they play a role in the regulation of the rhythmic release of hormones. Thus, we speculate that a subset of genes that are important in gastrointestinal motility such as neurotransmitter enzymes and neurotransmitter receptors are under direct or indirect clock control. Indeed, healthy people have bowel movements during the day, frequently following awakening or following a meal but rarely during the night. In line with this observation are ambulatory pressure recordings from healthy individuals demonstrating maximal colonic activity during the day, especially following awakening and following a meal, and minimal activity during the night. ${ }^{6}$ A circadian rhythm was described for rectal motor complexes in fully ambulant subjects using anorectal pressure recordings as well. ${ }^{42}$ Thus, colonic activity follows a rhythmic pattern, with increased activity during the day suggestive of possible circadian regulation. Furthermore, disruption of the clock mechanism such as occurs with shift work has been associated with the development of gastrointestinal symptoms. ${ }^{9,10}$

In summary, clock genes are expressed in the murine intestine in a circadian manner. Clock genes can change their phase of expression in response to changes in the feeding cycle, indicating that feeding is an important cue for gastrointestinal clock gene expression. The predominant expression of clock genes in the epithelial cells and the myenteric plexus suggests a possible role for clock genes in the coordination of cell proliferation and motility.

\section{References}

1. Bell-Pedersen D, Cassone VM, Earnest DJ, et al. Circadian rhythms from multiple oscillators: lessons from diverse organisms. Nat Rev Genet 2005;6:544-556.

2. Cornelissen GKE, Halberg J, Halberg F. Toward the chronobiology and chronomics of the intestine. Elsevier, the Netherlands, 2002.

3. Hoogerwerf WA. Biologic clocks and the gut. Curr Gastroenterol Rep 2006;8:353-359.

4. Scheving LA. Biological clocks and the digestive system. Gastroenterology 2000;119:536-549.

5. Moore JG, Englert E Jr. Circadian rhythm of gastric acid secretion in man. Nature 1970;226:1261-1262.

6. Rao SS, Sadeghi P, Beaty J, et al. Ambulatory 24-hour colonic manometry in healthy humans. Am J Physiol Gastrointest Liver Physiol 2001;280:G629-G639.

7. Rhoads DB, Rosenbaum DH, Unsal $\mathrm{H}$, et al. Circadian periodicity of intestinal $\mathrm{Na}+$ /glucose cotransporter $1 \mathrm{mRNA}$ levels is transcriptionally regulated. J Biol Chem 1998;273:9510-9516.

8. Froy 0 , Chapnik N. Circadian oscillation of innate immunity components in mouse small intestine. Mol Immunol 2007;44:19641970.

9. Caruso CC, Lusk SL, Gillespie BW. Relationship of work schedules to gastrointestinal diagnoses, symptoms, and medication use in auto factory workers. Am J Ind Med 2004;46:586-598.

10. Vener KJ, Szabo S, Moore JG. The effect of shift work on gastrointestinal (GI) function: a review. Chronobiologia 1989;16:421439.

11. Moore RY, Speh JC, Leak RK. Suprachiasmatic nucleus organization. Cell Tissue Res 2002;309:89-98.

12. Cassone VM, Stephan FK. Central and peripheral regulation of feeding and nutrition by the mammalian circadian clock: implications for nutrition during manned space flight. Nutrition 2002;18: 814-819.

13. Brown SA, Schibler U. The ins and outs of circadian timekeeping. Curr Opin Genet Dev 1999;9:588-594.

14. Nakao K, Takahashi T, Utsunomiya J, et al. Extrinsic neural control of nitric oxide synthase expression in the myenteric plexus of rat jejunum. J Physiol 1998;507:549-560.

15. Shimamura M, Garcia JM, Prough DS, et al. Laser capture microdissection and analysis of amplified antisense RNA from distinct cell populations of the young and aged rat brain: effect of traumatic brain injury on hippocampal gene expression. Brain Res Mol Brain Res 2004;122:47-61. 
16. Bingham C, Arbogast B, Guillaume GC, et al. Inferential statistical methods for estimating and comparing cosinor parameters. Chronobiologia 1982;9:397-439.

17. Yamamoto $\mathrm{T}$, Nakahata $\mathrm{Y}$, Soma $\mathrm{H}$, et al. Transcriptional oscillation of canonical clock genes in mouse peripheral tissues. BMC Mol Biol 2004;5:18.

18. Davidson AJ, Poole AS, Yamazaki S, et al. Is the food-entrainable circadian oscillator in the digestive system? Genes Brain Behav 2003;2:32-39.

19. Hara R, Wan K, Wakamatsu $\mathrm{H}$, et al. Restricted feeding entrains liver clock without participation of the suprachiasmatic nucleus. Genes Cells 2001;6:269-278.

20. Damiola F, Le Minh N, Preitner N, et al. Restricted feeding uncouples circadian oscillators in peripheral tissues from the central pacemaker in the suprachiasmatic nucleus. Genes Dev 2000;14:2950-2961.

21. Stokkan KA, Yamazaki S, Tei H, et al. Entrainment of the circadian clock in the liver by feeding. Science 2001;291:490-493.

22. Zvonic S, Ptitsyn AA, Conrad SA, et al. Characterization of peripheral circadian clocks in adipose tissues. Diabetes 2006;55:962970.

23. Miki $\mathrm{H}$, Yano $\mathrm{M}$, Iwanaga $\mathrm{H}$, et al. Total parenteral nutrition entrains the central and peripheral circadian clocks. Neuroreport 2003;14:1457-1461.

24. Canaple L, Rambaud J, Dkhissi-Benyahya O, et al. Reciprocal regulation of brain and muscle Arnt-like protein 1 and peroxisome proliferator-activated receptor alpha defines a novel positive feedback loop in the rodent liver circadian clock. Mol Endocrinol 2006;20:1715-1727.

25. Hirota T, Fukada Y. Resetting mechanism of central and peripheral circadian clocks in mammals. Zoolog Sci 2004;21:359368.

26. Oishi K, Kasamatsu M, Ishida N. Gene- and tissue-specific alterations of circadian clock gene expression in streptozotocin-induced diabetic mice under restricted feeding. Biochem Biophys Res Commun 2004;317:330-334.

27. Wakamatsu H, Yoshinobu Y, Aida R, et al. Restricted feedinginduced anticipatory activity rhythm is associated with a phaseshift of the expression of mPer1 and mPer2 mRNA in the cerebral cortex and hippocampus but not in the suprachiasmatic nucleus of mice. Eur J Neurosci 2001;13:1190-1196.

28. Gooley JJ, Schomer A, Saper CB. The dorsomedial hypothalamic nucleus is critical for the expression of food-entrainable circadian rhythms. Nat Neurosci 2006;9:398-407.

29. Mieda M, Williams SC, Richardson JA, et al. The dorsomedial hypothalamic nucleus as a putative food-entrainable circadian pacemaker. Proc Natl Acad Sci U S A 2006;103:12150-12155.

30. Bando H, Nishio T, van der Horst GT, et al. Vagal regulation of respiratory clocks in mice. J Neurosci 2007;27:4359-4365.

31. Buijs RM, Chun SJ, Niijima A, et al. Parasympathetic and sympathetic control of the pancreas: a role for the suprachiasmatic nucleus and other hypothalamic centers that are involved in the regulation of food intake. J Comp Neurol 2001;431:405-423.

32. Tavakkolizadeh A, Ramsanahie A, Levitsky LL, et al. Differential role of vagus nerve in maintaining diurnal gene expression rhythms in the proximal small intestine. J Surg Res 2005;129: 73-78.

33. Goo RH, Moore JG, Greenberg E, et al. Circadian variation in gastric emptying of meals in humans. Gastroenterology 1987; 93:515-518.

34. Panda S, Antoch MP, Miller BH, et al. Coordinated transcription of key pathways in the mouse by the circadian clock. Cell 2002; 109:307-320.

35. Buchi KN, Moore JG, Hrushesky WJ, et al. Circadian rhythm of cellular proliferation in the human rectal mucosa. Gastroenterology 1991;101:410-415.

36. Scheving LE, Tsai TH, Scheving LA. Chronobiology of the intestinal tract of the mouse. Am J Anat 1983;168:433-465.

37. Matsuo T, Yamaguchi S, Mitsui S, et al. Control mechanism of the circadian clock for timing of cell division in vivo. Science 2003;302:255-259.

38. Levi F, Zidani R, Misset JL. Randomised multicentre trial of chronotherapy with oxaliplatin, fluorouracil, and folinic acid in metastatic colorectal cancer. International Organization for Cancer Chronotherapy. Lancet 1997;350:681-686.

39. Haus E. Chronobiology of the mammalian response to ionizing radiation. Potential applications in oncology. Chronobiol Int 2002; 19:77-100.

40. Schernhammer ES, Laden F, Speizer FE, et al. Night-shift work and risk of colorectal cancer in the Nurses' Health Study. J Nat Cancer Inst 2003;95:825-828.

41. Grundy D, Al-Chaer ED, Aziz Q, et al. Fundamentals of neurogastroenterology: basic science. Gastroenterology 2006;130:13911411.

42. Auwerda JJ, Bac DJ, Schouten WR. Circadian rhythm of rectal motor complexes. Dis Colon Rectum 2001;44:1328-1332.

Received December 20, 2006. Accepted June 28, 2007.

Address requests for reprints to: Willemijntje A. Hoogerwerf, MD, University of Michigan, Department of Internal Medicine, Division of Gastroenterology, VA Ann Arbor Healthcare System, 2215 Fuller Road, Ann Arbor, Michigan 48105. e-mail willemij@umich.edu; fax (734) 845-3237.

Supported by R21 DK074477-01A1 (to W.A.H.)

The authors thank Debbie R. Kennedy Boone and Jeanna M. Garcia for technical assistance with the laser capture microdissection and Joshua R. Scott for technical assistance with the PCR; Stephen A. Ernst, PhD, and Bradley B. Nelson (Michigan Gastrointestinal Peptide Research Center) for technical assistance with the slide preparation; Yuanxu Lu for the completion of the vagotomies; and John Wiley, MD, for critical review of the manuscript.

Conflict of interest: None of the authors have a conflict of interest to disclose. 